Vol. 15, n² | 2011

Varia

\title{
Jean-Noël Luc (dir.), Soldats de la loi. La gendarmerie
} au $X X^{e}$ siècle

Paris, PUPS, 2010, 534 pp. , ISBN 978-2-84050-693-5.

Jonas Campion

\section{(2) OpenEdition}

\section{Journals}

Édition électronique

URL : https://journals.openedition.org/chs/1306

DOI : $10.4000 /$ chs. 1306

ISSN : 1663-4837

\section{Éditeur}

Librairie Droz

Édition imprimée

Date de publication : 1 décembre 2011

Pagination : 157-161

ISSN : 1422-0857

\section{Référence électronique}

Jonas Campion, « Jean-Noël Luc (dir.), Soldats de la loi. La gendarmerie au XXe siècle », Crime, Histoire \& Sociétés / Crime, History \& Societies [En ligne], Vol. 15, n² | 2011, mis en ligne le 08 mars 2013, consulté le 23 mars 2022. URL : http://journals.openedition.org/chs/1306 ; DOI : https://doi.org/ 10.4000/chs.1306

Ce document a été généré automatiquement le 23 mars 2022.

(c) Droz 


\section{Jean-Noël Luc (dir.), Soldats de la loi. La gendarmerie au $\mathrm{XX}^{e}$ siècle}

Paris, PUPS, 2010, 534 pp. , ISBN 978-2-84050-693-5.

Jonas Campion

\section{RÉFÉRENCE}

Jean-Noël Luc (dir.), Soldats de la loi. La gendarmerie au XXe siècle, Paris, PUPS, 2010, 534 pp. , ISBN 978-2-84050-693-5.

1 Dix ans après la signature d'une convention de recherche entre ce qui était alors le Service historique de la gendarmerie nationale (devenu depuis le département gendarmerie du Service historique de la défense) et l'Université Paris IV-Sorbonne, Jean-Noël Luc, pionnier et principal animateur du chantier de l'histoire de la gendarmerie en France, publie une synthèse collective sur l'histoire de cette institution $\mathrm{au} \mathrm{XXe}$ siècle. Elle constitue un exemple supplémentaire du dynamisme éditorial du domaine de recherches "gendarmerie ", reléguant définitivement dans l'ombre l'egohistoire, l'histoire à charge, ou l'utilisation désincarnée des archives de l'arme, sans s'intéresser à leurs auteurs ou au contexte de leur production. Cette synthèse fait suite aux publications sur la gendarmerie au XIX ${ }^{\mathrm{e}}$ siècle (2002), sur les «figures de gendarmes » (2003) et à l'imposant guide de recherche sur l'histoire de la maréchaussée et de la gendarmerie $(2004)^{1}$. Elle s'intègre également dans la continuité de la dynamique éditoriale menée par la jeune Société d'histoire et du patrimoine de la gendarmerie (SNHPG), ou de diverses autres initiatives récentes, comme la publication des travaux de la rencontre sur la gendarmerie et la transition politique au XIX ${ }^{\mathrm{e}}$ siècle, organisée par Marc Bergère à Rennes (2007)².

2 À certains égards, cette publication constitue un bilan et la fin d'un premier cycle de recherches sur l'institution. Maintenant que les cadres principaux en sont dressés, il revient aux (jeunes) historiens qui s'y impliquent, d'en renouveler les questionnements dans les années à venir, pour garantir la pérennité et le succès de la problématique. 
Dans ce but, il faut encore multiplier les sources mobilisées, les niveaux d'échelle ou les angles d'approche du fait gendarmique. D'ores et déjà, deux grands projets viennent immédiatement à l'esprit, à la lecture de cette synthèse: il convient d'abord d'incarner plus encore le gendarme contemporain, de lui rendre son visage en tant qu'homme (puis femme), avec une famille, des intérêts, des logiques personnelles. Ensuite, il reste à questionner les influences et les relations entre gendarmes et gendarmeries, dans une perspective transnationale.

3 Si l'objectif initial de cette entreprise éditoriale était de mettre à la disposition de la communauté scientifique les actes d'un colloque tenu à Paris en 2003 sur la gendarmerie au $\mathrm{XX}^{\mathrm{e}}$ siècle, le temps écoulé a permis d'aller bien plus loin. Car, l'ouvrage n'est pas qu'une simple édition des actes de cette rencontre. Des articles supplémentaires aux communications présentées lors du colloque y sont publiés (celui d'Anne Geesen sur la féminisation de la gendarmerie par exemple), illustrant ainsi les développements les plus récents des prismes d'analyse de l'histoire de l'institution.

Ensuite, l'introduction de Jean-Noël Luc apporte une plus-value évidente au livre, de par la richesse des analyses qu'elle aborde. Elle propose une longue relecture historiographique des études déjà menées sur la gendarmerie, des chantiers en cours et de ceux qui restent à explorer. Jean-Noël Luc y dresse également une synthèse de l'évolution de l'institution et des enjeux sociopolitiques qu'elle incarne dans la France des Troisième, Quatrième et Cinquième Républiques. Il en discute enfin la chronologie et les rythmes institutionnels spécifiques. Selon la perspective adoptée, le $\mathrm{XX}^{\mathrm{e}}$ siècle de la gendarmerie débuterait avec l'adoption du décret organique de 1903 ou avec la Première Guerre mondiale, pour s'achever soit en 1988 avec un mouvement social inédit au sein du corps, soit en 2008 , avec le vote de la loi rattachant la gendarmerie au ministère de l'Intérieur. Au regard introductif de l'historien, répond la conclusion du sociologue. François Dieu, précurseur de la sociologie gendarmique française, revient sur "quelques réflexions sur la gendarmerie du début du XXI" siècle», inscrivant son propos dans la récente accélération des réformes au sein de l'institution depuis son entrée dans le giron du ministère de l'Intérieur. La lecture de cette contribution souligne la parenté, mais aussi les ruptures de la gendarmerie actuelle avec l'arme des XIX et $\mathrm{XX}^{\mathrm{e}}$ siècles. Plus exactement, elle confirme la persistance sur le temps long d'un champ de débats et enjeux autour de chaque police nationale et militaire (par exemple, le degré de militarité, la question de la polyvalence, l'insertion territoriale, la place au sein de l'appareil étatique de maintien de l'ordre, la relation au pouvoir exécutif...), qui constitue autant de clés de lecture indispensables à appréhender pour le chercheur.

Bilans des contributions dont ils en dressent les lignes de forces, ces deux textes confirment la nécessité pour l'historien de sans cesse réfléchir aux chronologies, ainsi qu'aux facteurs des continuités et ruptures qui traversent les institutions de régulations sociales dans les sociétés occidentales. Il s'agit là de préalables indispensables, pour qui s'intéresse aux rôles, fonctions et missions de ces institutions dans l'espace public, sans tomber dans un propos qui ne prendrait pas en compte les interactions réciproques entre la gendarmerie et le contexte politique ou social où elle évolue. Car, si par son action préventive et répressive, l'arme est révélatrice de la société qu'elle encadre, de ses peurs et de ses désordres, elle en représente également un acteur majeur, dont les prises de position institutionnelles d'abord, individuelles ensuite, ne sont pas à négliger (voir à ce propos, l'article de Jacques Frémeaux relatif à 
l'attitude de la gendarmerie dans la lutte contre l'OAS ou celui de Jean-François Nativité sur l'engagement des gendarmes au sein des FFL).

6 Enfin, la richesse des annexes (chronologie, organigrammes, tableaux d'effectifs, bibliographie commentée), qui actualisent et complètent les données équivalentes présentées dans le Guide de recherche de 2004, constituent autant d'éléments dépassant la simple édition d'actes de colloque. Revers de la médaille du temps qui s'est écoulé, certains des articles auraient sans doute mérité une mise à jour parfois plus approfondie - notamment en termes de nuances de propos tenus dans le corps du texte, à partir de certains travaux universitaires récents par exemple - que la simple révision bibliographique de notes en bas de page...

7 Soldats de la loi concerne exclusivement la gendarmerie française, contrairement à l'étude précédemment consacrée au XIX ${ }^{e}$ siècle, où l'exportation (imposition?) du modèle français de gendarmerie était détaillée et discutée. C'est un (léger) regret, d'autant plus que la question de l'acculturation gendarmique du $\mathrm{XX}^{e}$ siècle est un sujet qui reste encore largement méconnu. Si la problématique internationale se développera dans les années à venir, des premières hypothèses ou études de cas auraient d'ores et déjà pu être utiles dans ce volume. La question a pris ces dernières années une acuité nouvelle, avec le renouveau de la coopération policière internationale, notamment instaurée au niveau européen. De plus, comme les auteurs l'illustrent avec justesse, la gendarmerie française se caractérise également par un haut degré d'internationalisation lors de ses missions: outre les guerres mondiales ou sa participation aux processus de décolonisation, les engagements récents de la gendarmerie l'amènent à participer, au sein d'instances internationales ou dans le cadre de relations bilatérales d'État à État, à des missions de maintien de l'ordre ou de formation de police (Afghanistan, Kosovo...). Enfin, les gendarmes, dans leurs missions de police militaire, constituent la prévôté de toute opération extérieure de l'armée. Malgré tout, les recherches ici présentées constituent des fondations solides pour une comparaison et un croisement des gendarmeries (extra-) européennes. Encore à écrire, cette approche transnationale doit à la fois s'intéresser aux influences réciproques qui traversent les armes en termes d'organisation, d'esprit de corps, de matériels, de doctrines ou de pratiques mais doit aussi envisager la place de l'institution gendarmerie au sein de chaque appareil étatique.

Car, si l'on compare la situation de la gendarmerie française à celle de ses voisines les plus proches (Belgique et Pays-Bas notamment), il est frappant de constater combien le modèle gendarmique n'y est que peu contesté ou discuté au cours du XX $\mathrm{XX}^{\mathrm{e}}$ siècle (du moins, jusqu'il y a une dizaine d'années). Ses fondamentaux restent majoritairement identiques au long des décennies. Au contraire, dès l'entre-deux-guerres, l'opportunité d'une police nationale d'identité militaire est largement mise en débat aux Pays-Bas et ses fonctions limitées dans l'après-guerre au profit d'une police nationale civile. La gendarmerie belge, confirmée par une loi de 1957 comme première force de police nationale est accusée, dès les années 1970, d'être un «État dans l'État » échappant de plus en plus au contrôle de ses autorités de tutelle. Elle fait dès lors l'objet d'une crise de légitimité qui, dans un contexte larvé de guerre des polices et de multiplication des dysfonctionnements en matière d'ordre ou de justice, aboutit à sa démilitarisation en 1992 et à la refonte complète de l'appareil policier belge en 1998 .

9 Rapidement dressé, ce constat éclaire la nécessité d'étudier les relations des armes à la chose politique, et la relation des politiques aux armes. Car, ces différences s'expliquent 
notamment par le mode de gouvernement en cours dans chaque pays. Notamment, le scrutin proportionnel en vigueur en Belgique ou aux Pays-Bas débouche nécessairement sur la constitution de gouvernements de coalition, multipliant la possibilité de voir le corps dépendre de tutelles politiquement opposées. Celui-ci peut alors devenir enjeu de tensions, mais il peut également profiter de l'espace de liberté que lui laissent des désaccords politiques. On le voit, tout au long du siècle, la question est capitale. Elle mérite de plus amples développements à l'échelle de l'ensemble des gendarmeries européennes, tant elle interroge le fonctionnement démocratique de la société contemporaine.

Les 28 contributions sont structurées en six parties chronologiques, qui nous amènent de la Belle Époque au mouvement social de 1988-1989 où les gendarmes et leurs familles dénoncèrent par voie de presse et par voie épistolaire, leur malaise face à leurs conditions de vie. Dans ce vaste panorama, la Seconde Guerre mondiale et la décolonisation s'érigent en événements charnières entre le premier et le second vingtième siècle du corps. Le constat qui ressort de ce découpage équilibré est évident: c'est la mise en valeur du rôle «d'homme-orchestre » des gendarmes dans la société française de par la variété des missions qui les occupent, entre force militaire de police et force de police militaire. Les gendarmes sont successivement ou simultanément militaires et/ou combattants (Première et Seconde Guerres mondiales, Algérie, Indochine...), agents de l'ordre, agents de (dé)colonisation, agent de souveraineté (Corse, Mayotte ou, dans une vaine tentative, en France occupée), agents administratifs ou agents de sécurité (la mission de police de la route, dont l'importance ne fait que croître). Les contributions soulignent également, au gré des études de cas, l'insertion parfois difficile du corps au sein des enjeux sociaux, militaires, politiques ou d'ordre qui ont traversé la France, les colonies ou l'Outre-mer tout au long du XXe siècle, ainsi que les nécessaires efforts identitaires et d'adaptation qu'il doit fournir face à une société en pleine mutation. Ces transformations, d'un État social à un État-providence, obligent l'arme et ses autorités à s'interroger sur sa militarité, son rôle dans la défense (intérieure) du territoire, sa mobilité, ou à adapter les réponses qu'il apporte à l'urbanisation ou aux progrès des moyens techniques et de communication. Les réflexions proposées sur ces multiples questions par la trentaine d'auteurs sont stimulantes. S'il est possible de soulever un petit bémol, c'est le focus parfois exclusivement gendarmique abordé dans les propos. Certes, l'exercice d'élargissement est difficile au vu de la faible place disponible dans une contribution de ce type. Pourtant, tant dans les sources mobilisées que dans les conclusions adoptées, il serait parfois intéressant d'ouvrir plus la focale, pour réintégrer l'exposé sur l'arme dans une perspective sociopolitique plus générale de l'évolution société française.

11 Comme nous l'avons déjà signalé, les nécessaires réflexions transversales aux points de vue proposés dans les différentes contributions sont dressées, dans une perspective de longue durée, dans l'introduction et la conclusion générale. À cet égard, sur l'ensemble du livre, l'équilibre entre le micro et le macro est bien réalisé, entre les particularités locales, les cas représentatifs et les tendances lourdes qui composent l'histoire de cette force centralisée de police. Bien sûr, il est toujours possible de regretter l'absence de tel ou tel sujet au sein du corpus de textes publiés. Des questions comme l'attitude de la gendarmerie face au front populaire, ou son rôle et son expertise dans le maintien de l'ordre sur le long terme auraient pu être intéressantes. Mais il ne s'agit là que de détails. Par la multiplicité des focus chronologiques, géographiques, thématiques abordés, c'est la pluralité et la diversité des aspects du métier gendarmique sur le long 
$\mathrm{XX}^{\mathrm{e}}$ siècle et les évolutions qui le traverse, qu'illustre avec brio le livre de Jean-Noël Luc et de sa «brigade d'auteurs ».

Pour conclure, nous ne pouvons que souligner les apports significatifs de cette initiative à l'étude des pratiques et fonctions de police dans les sociétés contemporaines. Les quelques remarques que nous venons de relever, loin d'être des critiques, veulent plutôt souligner les nombreuses contributions de ce livre à l'historiographie policière, tout en soulignant les questions qu'il reste à aborder. Sans aucun doute, cette synthèse nationale est particulièrement utile. Car il manque encore - tant en France qu'à l'étranger ${ }^{3}$ - de perspectives historiques globales sur la gendarmerie sur le long $\mathrm{XX}^{\mathrm{e}}$ siècle et ses conditions particulières de guerres, de crises ou de globalisation. Autant la gendarmerie du XIXe siècle est bien connue, notamment son rôle de légitimation dans le processus de formation des États-nations, par ses fonctions d'agent centralisateur et d'acceptation du pouvoir national, autant les perspectives historiques sur les corps durant le $\mathrm{XX}^{\mathrm{e}}$ siècle étaient restées, jusqu'à présent, trop parcellaires et limitées. Soldats de la loi,parce qu'il éclaire les spécificités du rôle de la gendarmerie dans l'appareil militaire, de justice, ou de maintien de l'ordre en France,comble donc un vide criant de la recherche. Fort de ces apports, il convient maintenant, comme nous l'avons déjà proposé, d'internationaliser le propos, pour discuter, pour le $\mathrm{XX}^{\mathrm{e}}$ siècle, la cohabitation, les concurrences et les acculturations des modèles et systèmes policiers et gendarmiques dans les sociétés européennes, depuis la Belle Époque jusqu'aux nouveaux enjeux nationaux et internationaux (terrorisme, cybercriminalité, ...) de la « crise sécuritaire » des dernières décennies.

\section{NOTES}

1. Jean-Noël Luc (dir.), Gendarmerie, État et Société au XIX ${ }^{e}$ siècle, Paris, Publications de la Sorbonne, 2002; Jean-Noël Luc (dir.), Sociétés \& Représentations, $\mathrm{n}^{\circ} 16$, septembre 2003, Figures de gendarmes; Jean-Noël Luc (dir.), Histoire de la Maréchaussée et de la gendarmerie. Guide de recherches, MaisonsAlfort, SHGN, 2004.

2. Voir la revue Force publique ou le numéro spécial de la revue Annales de Bretagne et des pays de l'Ouest, t.114, $n^{\circ} 2$, juin 2007, sous la direction de Marc Bergère (Rennes 2).

3. À l'exception du récent projet mené aux Pays-Bas par Cyrille Fijnaut et son équipe. Voir Cyrille Fijnaut, De geschiedenis van de Nederlandse Politie. Een staatinstelling in de maalstroom van de geschiedenis, Amsterdam, Boom, 2007; Id., A history of the Dutch Police, Amsterdam, Boom, 2008 ou Jos Smeets, De Geschiedenis van de Nederlandse Politie. Verdeelheid en eenheid in het rijkspolitieapparaat, Amsterdam, Boom, 2007. 


\section{AUTEURS}

\section{JONAS CAMPION}

Jonas.campion@uclouvain.be 